\title{
A review and meta-analysis of the effect of weight loss on all-cause mortality risk
}

\author{
Mary Harrington $^{1}$, Sigrid Gibson ${ }^{2}$ and Richard C. Cottrell ${ }^{3 *}$ \\ ${ }^{1}$ The Sugar Bureau, London WC2B 5JJ, UK \\ ${ }^{2}$ Sig-Nurture Ltd, Guildford, Surrey GU1 2TF, UK \\ ${ }^{3}$ World Sugar Research Organisation, London SWIV 3LX, UK
}

\begin{abstract}
Overweight and obesity are associated with increased morbidity and mortality, although the range of body weights that is optimal for health is controversial. It is less clear whether weight loss benefits longevity and hence whether weight reduction is justified as a prime goal for all individuals who are overweight (normally defined as BMI $>25 \mathrm{~kg} / \mathrm{m}^{2}$ ). The purpose of the present review was to examine the evidence base for recommending weight loss by diet and lifestyle change as a means of prolonging life. An electronic search identified twenty-six eligible prospective studies that monitored subsequent mortality risk following weight loss by lifestyle change, published up to 2008. Data were extracted and further analysed by meta-analysis, giving particular attention to the influence of confounders. Moderator variables such as reason for weight loss (intentional, unintentional), baseline health status (healthy, unhealthy), baseline BMI (normal, overweight, obese), method used to estimate weight loss (measured weight loss, reported weight loss) and whether models adjusted for physical activity (adjusted data, unadjusted data) were used to classify subgroups for separate analysis. Intentional weight loss per se had a neutral effect on all-cause mortality (relative risk (RR) $1.01 ; P=0 \cdot 89$ ), while weight loss which was unintentional or ill-defined was associated with excess risk of 22 to $39 \%$. Intentional weight loss had a small benefit for individuals classified as unhealthy (with obesity-related risk factors) (RR 0.87 (95\% CI 0.77, 0.99); $P=0.028$ ), especially unhealthy obese (RR 0.84 (95\% CI $0.73,0.97) ; P=0.018)$, but appeared to be associated with slightly increased mortality for healthy individuals (RR $1.11(95 \%$ CI 1.00, 1.22); $P=0.05)$, and for those who were overweight but not obese (RR 1.09 (95\% CI 1.02, 1.17); $P=0.008$ ). There was no evidence for weight loss conferring either benefit or risk among healthy obese. In conclusion, the available evidence does not support solely advising overweight or obese individuals who are otherwise healthy to lose weight as a means of prolonging life. Other aspects of a healthy lifestyle, especially exercise and dietary quality, should be considered. However, well-designed intervention studies are needed clearly to disentangle the influence of physical activity, diet strategy and body composition, in order to define appropriate advice to those populations that might be expected to benefit.
\end{abstract}

Weight loss: All-cause mortality: Meta-analyses

\section{Introduction}

Weight loss has been reported to result in several health benefits, such as significant improvements in CVD risk factors (blood pressure, lipid profiles, glucose tolerance $)^{(1,2)}$. It may therefore be reasonable to expect that weight loss would lead to decreased mortality in the long term. Indeed, this seems to be the case in obese individuals with serious medical complications ${ }^{(3,4)}$ or when substantial weight loss has followed surgical procedures ${ }^{(5)}$. However, the long-term effects of more moderate degrees of weight loss for those who are not severely obese and do not have co-morbidities are unclear. Many prospective studies show conflicting results, while some recent studies indicate either excess ${ }^{(6-9)}$ or unchanged mortality ${ }^{(10)}$ following weight loss. Reviews of the data suggest that inconsistent results might be due to failure to control for known confounding factors (for example, underlying disease, intention to lose weight $)^{(11,12)}$ while also noting that many of the existing studies were not specifically designed to test the hypothesis that weight loss increases or decreases

Abbreviation: RR, relative risk.

* Corresponding author: Dr Richard C. Cottrell, fax +1 207834 4137, email rcottrell@wsro.org 


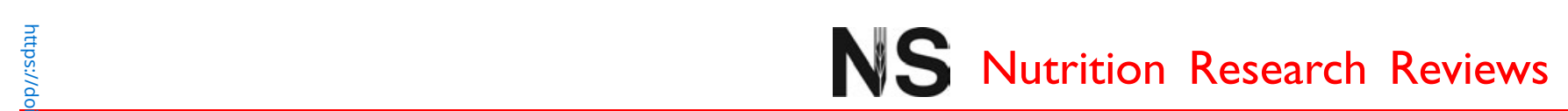

Table 1. Prospective studies of weight loss and mortality*

\begin{tabular}{|c|c|c|c|c|c|c|c|c|c|c|c|}
\hline $\begin{array}{l}\text { Study } \\
\text { reference }\end{array}$ & Population & $\begin{array}{l}\text { Referent } \\
\text { group }\end{array}$ & $\begin{array}{l}\text { Weight } \\
\text { loss } \\
\text { subgroup }\end{array}$ & $\begin{array}{l}\text { Sample } \\
\text { size }(n)\end{array}$ & $\begin{array}{l}\text { Mean } \\
\text { baseline } \\
\text { age } \\
\text { and/or } \\
\text { range } \\
\text { (years) }\end{array}$ & $\begin{array}{l}\text { Baseline } \\
\text { weight } \\
(\mathrm{kg}) \text { or } \\
\mathrm{BMI} \\
\left(\mathrm{kg} / \mathrm{m}^{2}\right)\end{array}$ & $\begin{array}{l}\text { Weight } \\
(\mathrm{kg}) \text { or } \\
\mathrm{BMI} \text { loss } \\
\left(\mathrm{kg} / \mathrm{m}^{2}\right)\end{array}$ & $\begin{array}{l}\text { Data } \\
\text { adjustments }\end{array}$ & $\begin{array}{l}\text { Relative } \\
\text { risk }\end{array}$ & $95 \% \mathrm{Cl}$ & Exclusions \\
\hline \multirow[t]{3}{*}{$\begin{array}{l}\text { Harris et al. } \\
(1988)^{(38)}\end{array}$} & \multirow{3}{*}{$\begin{array}{l}\text { Apparently } \\
\text { healthy men } \\
\text { and women } \\
\text { Framingham } \\
\text { Heart Study } \\
\text { Change in } \\
\text { BMI from age } \\
55 \text { to } 65 \text { years }\end{array}$} & \multirow[t]{3}{*}{$\begin{array}{l}0-9 \% \text { BMl } \\
\text { gain }\end{array}$} & \multirow[t]{2}{*}{$\begin{array}{l}\text { Men: weight } \\
\text { loss intention } \\
\text { unspecified }\end{array}$} & \multirow[t]{2}{*}{-} & \multirow[t]{2}{*}{$55-65$} & \multirow[t]{2}{*}{-} & $\geq 10 \% \mathrm{BMI}$ & \multirow[t]{3}{*}{ Baseline weight } & 1.9 & $1 \cdot 1,3 \cdot 2$ & \multirow[t]{3}{*}{$\begin{array}{l}\text { Smokers, } \\
\text { ex-smokers }\end{array}$} \\
\hline & & & & & & & $0-9 \% \mathrm{BMI}$ & & 1.4 & $1 \cdot 0,1 \cdot 9$ & \\
\hline & & & \multirow{2}{*}{$\begin{array}{l}\text { Women: weight } \\
\text { loss intention } \\
\text { unspecified } \\
\text { Men: weight } \\
\text { loss intention } \\
\text { unspecified }\end{array}$} & - & $55-65$ & - & $\begin{array}{l}\geq 10 \% \mathrm{BMI} \\
0-9 \% \mathrm{BMI}\end{array}$ & & $\begin{array}{l}1.8 \\
1.1\end{array}$ & $\begin{array}{l}1 \cdot 2,2 \cdot 6 \\
0 \cdot 8,1 \cdot 4\end{array}$ & \\
\hline \multirow[t]{13}{*}{$\begin{array}{l}\text { Pamuk et al. } \\
(1992)^{(39)}\end{array}$} & $\begin{array}{l}\text { Apparently } \\
\text { healthy men } \\
\text { and women }\end{array}$ & \multirow[t]{13}{*}{$\begin{array}{l}<5 \% \text { maxi- } \\
\text { mum } \\
\text { weight loss }\end{array}$} & & 68 & \multirow[t]{6}{*}{$45-74$} & $\begin{array}{c}26-<29 \\
\mathrm{~kg} / \mathrm{m}^{2}\end{array}$ & $6.5 \%$ weight & \multirow[t]{13}{*}{$\begin{array}{l}\text { Age, race, smoking } \\
\text { parity, pre-existing } \\
\text { illness, maximum } \\
\text { BMI }\end{array}$} & 1.7 & $1 \cdot 1,2 \cdot 7$ & \multirow[t]{13}{*}{$\begin{array}{l}\text { Deaths in } \\
\text { first } 5 \text { years }\end{array}$} \\
\hline & \multirow[t]{12}{*}{ NHANES I } & & & 279 & & $<26 \mathrm{~kg} / \mathrm{m}^{2}$ & $9.4 \%$ weight & & 1.4 & $1 \cdot 0,1 \cdot 8$ & \\
\hline & & & & 97 & & $<26 \mathrm{~kg} / \mathrm{m}^{2}$ & $20.3 \%$ weight & & 2.4 & $1.7,3.5$ & \\
\hline & & & & $\begin{array}{l}1 / 2 \\
204\end{array}$ & & $\begin{array}{l}\geq 29 \mathrm{~kg} / \mathrm{m}^{2} \\
26-<29\end{array}$ & $\begin{array}{l}8.8 \% \text { weight } \\
9.7 \% \text { weight }\end{array}$ & & $\begin{array}{l}0.1 \\
0.8\end{array}$ & $\begin{array}{l}0.5,1 \cdot 0 \\
0.6,1 \cdot 1\end{array}$ & \\
\hline & & & & $\begin{array}{l}34 \\
68\end{array}$ & & $\begin{array}{l}\mathrm{kg} / \mathrm{m}^{2} \\
\geq 29 \mathrm{~kg} / \mathrm{m}^{2} \\
26-<29 \\
\mathrm{~kg} / \mathrm{m}^{2}\end{array}$ & $\begin{array}{l}18.1 \% \text { weight } \\
20 \% \text { weight }\end{array}$ & & $\begin{array}{l}0.8 \\
1.1\end{array}$ & $\begin{array}{l}0.4,1.5 \\
0.7,1.6\end{array}$ & \\
\hline & & & & 105 & & $<26 \mathrm{~kg} / \mathrm{m}^{2}$ & $23.2 \%$ weight & & 1.4 & $1 \cdot 0,2 \cdot 0$ & \\
\hline & & & $\begin{array}{l}\text { Women: weight } \\
\text { loss intention } \\
\text { unspecified }\end{array}$ & 38 & $45-74$ & $\begin{array}{c}26-<29 \\
\mathrm{~kg} / \mathrm{m}^{2}\end{array}$ & $6.6 \%$ weight & & 1.4 & $0.6,2.9$ & \\
\hline & & & & 223 & & $<26 \mathrm{~kg} / \mathrm{m}^{2}$ & $10.2 \%$ weight & & 1.6 & $1 \cdot 0,2 \cdot 6$ & \\
\hline & & & & 128 & & $<26 \mathrm{~kg} / \mathrm{m}^{2}$ & $20.3 \%$ weight & & & $1.3,3.6$ & \\
\hline & & & & 230 & & $\geq 29 \mathrm{~kg} / \mathrm{m}^{2}$ & $9.3 \%$ weight & & 1.0 & $0.7,1.4$ & \\
\hline & & & & 168 & & $\begin{array}{c}26-<29 \\
\mathrm{~kg} / \mathrm{m}^{2}\end{array}$ & $9.8 \%$ weight & & 1.5 & $1 \cdot 0,2 \cdot 1$ & \\
\hline & & & & $\begin{array}{l}97 \\
80\end{array}$ & & $\begin{array}{l}\geq 29 \mathrm{~kg} / \mathrm{m}^{2} \\
26-<29 \\
\mathrm{~kg} / \mathrm{m}^{2}\end{array}$ & $\begin{array}{l}20.9 \% \text { weight } \\
20.9 \% \text { weight }\end{array}$ & & $\begin{array}{l}1.4 \\
1.4\end{array}$ & $\begin{array}{l}0.9,2 \cdot 1 \\
0.9,2 \cdot 1\end{array}$ & \\
\hline & & & & 151 & & $<26 \mathrm{~kg} / \mathrm{m}^{2}$ & $25.5 \%$ weight & & 1.9 & $1 \cdot 3,2 \cdot 6$ & \\
\hline \multirow{2}{*}{$\begin{array}{l}\text { Lee \& } \\
\text { Paffenbar- } \\
\text { ger } \\
(1992)^{(1)}\end{array}$} & $\begin{array}{l}\text { Apparently } \\
\text { healthy men }\end{array}$ & \multirow[t]{2}{*}{$\begin{array}{l}\text { Weight } \\
\text { stable }\end{array}$} & \multirow[t]{2}{*}{$\begin{array}{l}\text { Men, weight } \\
\text { loss intention } \\
\text { unspecified }\end{array}$} & 1293 & 58 & $78 \mathrm{~kg}$ & $>5 \mathrm{~kg}$ weight & \multirow[t]{2}{*}{$\begin{array}{l}\text { Age, height, } \\
\text { smoking, physical } \\
\text { activity }\end{array}$} & 1.57 & $\begin{array}{c}1.34 \\
1.84\end{array}$ & \multirow[t]{2}{*}{$\begin{array}{l}\text { Baseline CVD, } \\
\text { cancer }\end{array}$} \\
\hline & $\begin{array}{l}\text { Harvard Alumni } \\
\text { Health Study }\end{array}$ & & & 2730 & 58 & $78 \mathrm{~kg}$ & $\begin{array}{c}1-5 \mathrm{~kg} \\
\text { weight }\end{array}$ & & 1.26 & $\begin{array}{l}1 \cdot 10, \\
1.46\end{array}$ & \\
\hline \multirow[t]{2}{*}{$\begin{array}{l}\text { Higgins et al. } \\
(1993)^{(40)}\end{array}$} & $\begin{array}{l}\text { Apparently } \\
\text { healthy men } \\
\text { and women }\end{array}$ & \multirow[t]{2}{*}{$\begin{array}{l}\text { No change } \\
\text { in BMI }\end{array}$} & Men & - & $45 \cdot 5$ & $27 \cdot 1 \mathrm{~kg} / \mathrm{m}^{2}$ & $0.52 \mathrm{~kg}$ & \multirow[t]{2}{*}{$\begin{array}{l}\text { Age, BMI, systolic } \\
\text { blood pressure, } \\
\text { cholesterol, } \\
\text { glucose intolerance, } \\
\text { left ventricular } \\
\text { hypertrophy, } \\
\text { smoking }\end{array}$} & 1.33 & $\begin{array}{l}1.06, \\
1.68\end{array}$ & \multirow[t]{2}{*}{$\begin{array}{l}\text { Deaths in } \\
\text { first } 4 \text { years }\end{array}$} \\
\hline & $\begin{array}{l}\text { Framingham } \\
\text { Study }\end{array}$ & & Women & - & $45 \cdot 8$ & $25 \cdot 7 \mathrm{~kg} / \mathrm{m}^{2}$ & $0.39 \mathrm{~kg}$ & & $1 \cdot 28$ & $\begin{array}{l}0.98, \\
1.68\end{array}$ & \\
\hline \multirow{3}{*}{$\begin{array}{l}\text { Chaturvedi \& } \\
\text { Fuller } \\
{(1995)^{(41)}}^{(19)}\end{array}$} & $\begin{array}{l}\text { NIDDM men } \\
\text { and women }\end{array}$ & \multirow[t]{3}{*}{ Weight stable } & $\begin{array}{l}\text { European men } \\
\text { and women }\end{array}$ & $\begin{array}{l}252 \text { (52 } \\
\quad \text { deaths) }\end{array}$ & $35-55$ & $<26 \mathrm{~kg} / \mathrm{m}^{2}$ & $>2 \mathrm{~kg} / \mathrm{m}^{2}$ & \multirow[t]{3}{*}{$\begin{array}{l}\text { Age, sex, duration of } \\
\text { diagnosed diabetes }\end{array}$} & 3.05 & $\begin{array}{c}1.26 \\
7.36\end{array}$ & - \\
\hline & WHO study & & $\begin{array}{l}\text { Weight loss } \\
\text { intention } \\
\text { unspecified }\end{array}$ & & $35-55$ & $\begin{array}{r}26-29 \\
\mathrm{~kg} / \mathrm{m}^{2}\end{array}$ & $>2 \mathrm{~kg} / \mathrm{m}^{2}$ & & 2.02 & $\begin{array}{l}1.00 \\
4.08\end{array}$ & \\
\hline & & & & & $35-55$ & $\geq 29 \mathrm{~kg} / \mathrm{m}^{2}$ & $>2 \mathrm{~kg} / \mathrm{m}^{2}$ & & 0.84 & $\begin{array}{l}0.40 \\
1.74\end{array}$ & \\
\hline
\end{tabular}




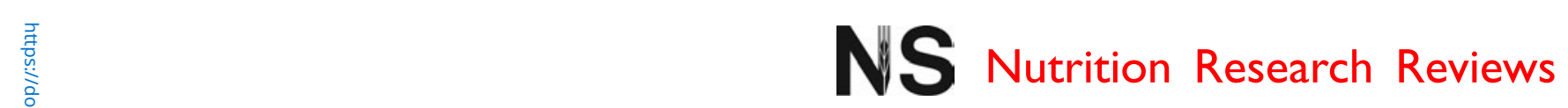

Table 1. Continued

\begin{tabular}{|c|c|c|c|c|c|c|c|c|c|c|c|}
\hline $\begin{array}{l}\text { Study } \\
\text { reference }\end{array}$ & Population & $\begin{array}{l}\text { Referent } \\
\text { group }\end{array}$ & $\begin{array}{l}\text { Weight } \\
\text { loss } \\
\text { subgroup }\end{array}$ & $\begin{array}{l}\text { Sample } \\
\text { size }(n)\end{array}$ & $\begin{array}{l}\text { Mean } \\
\text { baseline } \\
\text { age } \\
\text { and/or } \\
\text { range } \\
\text { (years) }\end{array}$ & $\begin{array}{l}\text { Baseline } \\
\text { weight } \\
(\mathrm{kg}) \text { or } \\
\mathrm{BMI} \\
\left(\mathrm{kg} / \mathrm{m}^{2}\right)\end{array}$ & $\begin{array}{l}\text { Weight } \\
(\mathrm{kg}) \text { or } \\
\mathrm{BMI} \text { loss } \\
\left(\mathrm{kg} / \mathrm{m}^{2}\right)\end{array}$ & $\begin{array}{l}\text { Data } \\
\text { adjustments }\end{array}$ & $\begin{array}{l}\text { Relative } \\
\text { risk }\end{array}$ & $95 \% \mathrm{Cl}$ & Exclusions \\
\hline \multirow[t]{2}{*}{$\begin{array}{c}\text { French et al. } \\
(1999)^{(47)}\end{array}$} & $\begin{array}{l}\text { Apparently } \\
\text { healthy } \\
\text { women }\end{array}$ & $\begin{array}{l}\text { Never } \geq 20 \text { lbs } \\
\text { weight loss }\end{array}$ & Intentional & 4300 & 66.6 & $30 \cdot 3 \mathrm{~kg} / \mathrm{m}^{2}$ & $\geq 9.1 \mathrm{~kg}$ & $\begin{array}{l}\text { Age, BMI, waist:hip } \\
\text { ratio, education, } \\
\text { marital status, } \\
\text { smoking, oestrogen } \\
\text { use, cancer, diabetes, } \\
\text { angina, stroke, heart } \\
\text { attack, hypertension }\end{array}$ & $1 \cdot 18$ & $\begin{array}{l}0.94 \\
1.48\end{array}$ & - \\
\hline & $\begin{array}{l}\text { lowa Women's } \\
\text { Health Study }\end{array}$ & & Unintentional & 5008 & 68.0 & $26 \cdot 1 \mathrm{~kg} / \mathrm{m}^{2}$ & $\geq 9 \cdot 1 \mathrm{~kg}$ & & 1.33 & $\begin{array}{c}1.13 \\
1.57\end{array}$ & \\
\hline \multirow[t]{7}{*}{$\begin{array}{l}\text { Williamson } \\
\text { et al. } \\
\quad(1999)^{(48)}\end{array}$} & $\begin{array}{l}\text { Healthy and } \\
\text { unhealthy } \\
\text { men }\end{array}$ & $\begin{array}{l}\text { No weight } \\
\text { change }\end{array}$ & Healthy & & & & & $\begin{array}{l}\text { Age, BMI, smoking, } \\
\text { education, alcohol } \\
\text { intake, physical, } \\
\text { activity, health } \\
\text { complaints }\end{array}$ & & & $\begin{array}{c}\mathrm{BMl}<27 \mathrm{~kg} / \mathrm{m}^{2}, \\
\text { non-Caucasian }\end{array}$ \\
\hline & $\begin{array}{l}\text { American } \\
\text { Cancer } \\
\text { Prevention } \\
\text { Study }\end{array}$ & & Unintentional & 1474 & $52 \cdot 0$ & $29.2 \mathrm{~kg} / \mathrm{m}^{2}$ & $3.2 \mathrm{~kg} / \mathrm{m}^{2}$ & & 1.04 & $\begin{array}{l}0.91 \\
1.19\end{array}$ & \\
\hline & & & $\begin{array}{l}\text { Intentional } \\
1-19 \text { lbs }\end{array}$ & 2834 & 51.5 & $29.0 \mathrm{~kg} / \mathrm{m}^{2}$ & $1.8 \mathrm{~kg} / \mathrm{m}^{2}$ & & 1.09 & $\begin{array}{l}0.98, \\
1.21\end{array}$ & \\
\hline & & & $\begin{array}{l}\text { Intentional } \\
\geq 20 \text { Ibs } \\
\text { Unhealthy }\end{array}$ & 2610 & 51.5 & $31.4 \mathrm{~kg} / \mathrm{m}^{2}$ & $4.5 \mathrm{~kg} / \mathrm{m}^{2}$ & & 1.07 & $\begin{array}{c}0.96, \\
1.20\end{array}$ & \\
\hline & & & Unintentional & 917 & 54.4 & $29.7 \mathrm{~kg} / \mathrm{m}^{2}$ & $4.2 \mathrm{~kg} / \mathrm{m}^{2}$ & & $1 \cdot 15$ & $\begin{array}{l}1.04 \\
1.27\end{array}$ & \\
\hline & & & $\begin{array}{c}\text { Intentional } \\
1-19 \mathrm{lbs}\end{array}$ & 1310 & 53.4 & $29.1 \mathrm{~kg} / \mathrm{m}^{2}$ & $1.9 \mathrm{~kg} / \mathrm{m}^{2}$ & & 1.01 & $\begin{array}{l}0.91 \\
1.12\end{array}$ & \\
\hline & & & $\begin{array}{l}\text { Intentional } \\
\geq 20 \mathrm{lbs}\end{array}$ & 2614 & 53.6 & $31.6 \mathrm{~kg} / \mathrm{m}^{2}$ & $4.9 \mathrm{~kg} / \mathrm{m}^{2}$ & & 1.02 & $\begin{array}{c}0.94 \\
1.11\end{array}$ & \\
\hline \multirow[t]{2}{*}{$\begin{array}{l}\text { Williamson } \\
\text { et al. } \\
(2000)^{(4)}\end{array}$} & $\begin{array}{l}\text { Unhealthy } \\
\text { men and } \\
\text { women }\end{array}$ & $\begin{array}{l}\text { No or unknown } \\
\text { weight change }\end{array}$ & Intentional & 1669 & 54.5 & $33.5 \mathrm{~kg} / \mathrm{m}^{2}$ & $5.8 \mathrm{~kg} / \mathrm{m}^{2}$ & $\begin{array}{l}\text { Age, sex, BMI, race, } \\
\text { smoking, education, } \\
\text { alcohol intake, } \\
\text { physical activity, } \\
\text { disease history }\end{array}$ & 0.75 & $\begin{array}{l}0.67 \\
0.84\end{array}$ & $\mathrm{BMI}<27 \mathrm{~kg} / \mathrm{m}^{2}$ \\
\hline & $\begin{array}{l}\text { American } \\
\text { Cancer } \\
\text { Prevention } \\
\text { Study }\end{array}$ & & Unintentional & 649 & 55.6 & $31.8 \mathrm{~kg} / \mathrm{m}^{2}$ & $5.9 \mathrm{~kg} / \mathrm{m}^{2}$ & & 0.98 & $\begin{array}{l}0.85 \\
1.13\end{array}$ & \\
\hline \multirow[t]{2}{*}{$\begin{array}{l}\text { Newman } \\
\text { et al. } \\
(2001)^{(49)}\end{array}$} & $\begin{array}{l}\text { Older men } \\
\text { and women }\end{array}$ & $\begin{array}{l}\text { Weight stable, } \\
\text { i.e. weight } \\
\text { within } \pm 5 \% \\
\text { of baseline }\end{array}$ & $\begin{array}{l}\text { Weight loss } \\
\text { intention } \\
\text { unspecified }\end{array}$ & 126 deaths & 77.4 & $27 \mathrm{~kg} / \mathrm{m}^{2}$ & $\begin{array}{l}\geq 5 \% \\
\text { weight }\end{array}$ & $\begin{array}{l}\text { Age, sex, race, } \\
\text { cognitive function, } \\
\text { medication, smoking, } \\
\text { waist circumference, } \\
\text { mobility impairment }\end{array}$ & 1.67 & $\begin{array}{l}1 \cdot 29, \\
2 \cdot 15\end{array}$ & $\begin{array}{l}\text { Living in an } \\
\text { institution, } \\
\text { wheelchair use, } \\
\text { cancer treat- } \\
\text { ment }\end{array}$ \\
\hline & & & & 62 deaths & 77.4 & & $\geq 5 \%$ & & 1.66 & 1.18, & Plus interim \\
\hline $\begin{array}{l}\text { Wanna- } \\
\text { methee } \\
\text { et al. } \\
(2002)^{(50)}\end{array}$ & $\begin{array}{l}\text { Apparently } \\
\text { healthy men }\end{array}$ & Weight stable & $\begin{array}{l}\text { Weight loss } \\
\text { intention } \\
\text { unspecified }\end{array}$ & 950 & $\begin{array}{r}40-59 \\
\text { years }\end{array}$ & $\begin{array}{l}26 \cdot 6 \\
\mathrm{~kg} / \mathrm{m}^{2}\end{array}$ & $\begin{array}{l}2 \cdot 11 \\
\mathrm{~kg} / \mathrm{m}^{2}\end{array}$ & $\begin{array}{l}\text { Age, social class, } \\
\text { smoking, physical } \\
\text { activity, BMI, CVD, } \\
\text { cancer, poor } \\
\text { health, diabetes }\end{array}$ & 1.34 & $\begin{array}{c}1.09 \\
1.63\end{array}$ & - \\
\hline
\end{tabular}




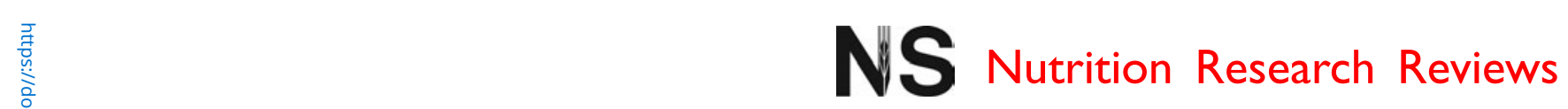

\begin{tabular}{|c|c|c|c|c|c|c|c|c|c|c|c|}
\hline \multirow[t]{3}{*}{$\begin{array}{l}\text { Gregg et al. } \\
(2003)^{(31)}\end{array}$} & $\begin{array}{l}\text { Overweight } \\
\text { or obese } \\
\text { men and } \\
\text { women }\end{array}$ & Weight stable & $\begin{array}{l}\text { Overall } \\
\text { (unspecified) }\end{array}$ & 1931 & $\begin{array}{l}\text { Over } 35 \\
\text { years } \\
\text { (mean } \\
54 \cdot 1)\end{array}$ & $\begin{array}{l}30 \cdot 8 \\
\mathrm{~kg} / \mathrm{m}^{2}\end{array}$ & $7 \mathrm{~kg}$ & $\begin{array}{l}\text { Age, sex, race, } \\
\text { smoking, education, } \\
\text { BMI, self-rated } \\
\text { health, diabetes, } \\
\text { acute and chronic } \\
\text { conditions, functional } \\
\text { limitations due } \\
\text { to CVD or cancer, } \\
\text { hospital bed days }\end{array}$ & 1.09 & $\begin{array}{l}0.90 \\
1.32\end{array}$ & $\begin{array}{l}\text { Baseline } \\
\mathrm{BMl}<25 \\
\mathrm{~kg} / \mathrm{m}^{2}\end{array}$ \\
\hline & $\begin{array}{l}\text { NHIS US } \\
\text { cohort }\end{array}$ & & Unintentional & 188 & - & - & $6.9 \mathrm{~kg}$ & & 1.31 & $\begin{array}{l}1.01, \\
1.70\end{array}$ & \\
\hline & $\begin{array}{l}\text { Retrospective } \\
\text { weight } \\
\text { change. } \\
\text { 9-year } \\
\text { follow-up }\end{array}$ & & Intentional & 827 & - & - & $7.1 \mathrm{~kg}$ & & 0.76 & $\begin{array}{c}0.60 \\
0.97\end{array}$ & \\
\hline \multirow[t]{3}{*}{$\begin{array}{l}\text { Gregg et al. } \\
(2004)^{(51)}\end{array}$} & $\begin{array}{l}\text { Unhealthy men } \\
\text { and } \\
\text { women } \\
\text { (diabetics) } \\
\text { from NHIS US } \\
\text { cohort } \\
\text { (9-year } \\
\text { follow-up) }\end{array}$ & Weight stable & $\begin{array}{l}\text { Overall } \\
\text { (unspecified) }\end{array}$ & 629 & - & $\begin{array}{l}33 \cdot 0 \\
\mathrm{~kg} / \mathrm{m}^{2}\end{array}$ & $6.80 \mathrm{~kg}$ & $\begin{array}{l}\text { Age, sex, race, BMI, } \\
\text { smoking, education, } \\
\text { self-rated health, } \\
\text { diabetes, medication, } \\
\text { length of disease, } \\
\text { functional limitation, } \\
\text { hypertension, stroke, } \\
\text { heart disease, retinal } \\
\text { disease, neuropathy, } \\
\text { hospital days, doctor } \\
\text { visits }\end{array}$ & $1 \cdot 19$ & $0.9,1 \cdot 47$ & $\begin{array}{l}\text { Baseline } \\
\mathrm{BMl}<25 \\
\mathrm{~kg} / \mathrm{m}^{2}\end{array}$ \\
\hline & & & Unintentional & 365 & - & - & $6.80 \mathrm{~kg}$ & & 1.58 & $\begin{array}{l}1.08 \\
2 \cdot 31\end{array}$ & \\
\hline & & & Intentional & 34 & - & - & $6.80 \mathrm{~kg}$ & & 0.83 & $\begin{array}{l}0.63, \\
1.08\end{array}$ & \\
\hline \multirow[t]{3}{*}{$\begin{array}{l}\text { Maru et al. } \\
(2004)^{(52)}\end{array}$} & Healthy women & $\begin{array}{l}\text { Weight stable, } \\
\text { i.e. }<5 \% \\
\text { weight } \\
\text { change }\end{array}$ & $\begin{array}{l}\text { Moderate weight } \\
\text { loss }\end{array}$ & 531 & $\begin{array}{c}50-66 \\
\text { years }\end{array}$ & $\begin{array}{l}\text { Median } \\
\text { BMl } \\
25.4 \mathrm{~kg} / \mathrm{m}^{2}\end{array}$ & $\begin{array}{l}5-9 \% \\
\text { weight }\end{array}$ & Age, smoking, BMI & $1 \cdot 14$ & $1 \cdot 1,1 \cdot 6$ & $\begin{array}{l}\text { Medication for } \\
\text { hypertension, } \\
\text { CVD, diabetes, } \\
\text { restriction diet }\end{array}$ \\
\hline & $\begin{array}{l}\text { DOM Dutch } \\
\text { cohort }\end{array}$ & & $\begin{array}{l}\text { Severe weight } \\
\text { loss }\end{array}$ & 108 & & $\begin{array}{l}\text { IQR 23. } \\
3-27.8 \\
\mathrm{~kg} / \mathrm{m}^{2}\end{array}$ & $\begin{array}{l}10-14 \% \\
\text { weight }\end{array}$ & & 0.9 & $0.5,1.4$ & \\
\hline & $\begin{array}{l}\text { Weight change } \\
\text { in } \\
\text { 1-year follow- } \\
\text { up } \\
\text { Median follow- } \\
\text { up } \\
17 \text { years }\end{array}$ & & $\begin{array}{l}\text { Extreme weight } \\
\text { loss }\end{array}$ & 43 & & & $\geq 15 \%$ weight & & 0.8 & $0.4,1 \cdot 8$ & \\
\hline $\begin{array}{l}\text { Diaz et al. } \\
(2005)^{(6)}\end{array}$ & $\begin{array}{l}\text { Apparently } \\
\text { healthy } \\
\text { men and } \\
\text { women } \\
\text { NHANES I and } \\
\text { follow-up }\end{array}$ & Weight stable & $\begin{array}{l}\text { Weight loss } \\
\text { intention } \\
\text { unspecified }\end{array}$ & 711 & 51.6 & $30.8 \mathrm{~kg} / \mathrm{m}^{2}$ & $5.55 \mathrm{~kg} / \mathrm{m}^{2}$ & $\begin{array}{l}\text { Age, sex, race, BMI, } \\
\text { smoking, health } \\
\text { status, poor health, } \\
\text { incapacitated }\end{array}$ & 3.36 & $\begin{array}{l}2 \cdot 47 \\
\quad 4.55\end{array}$ & $\begin{array}{l}\text { Diabetes, CVD, } \\
\text { cancer }\end{array}$ \\
\hline \multirow[t]{2}{*}{$\begin{array}{l}\text { Drøyvold } \\
\text { et al. } \\
(2005)^{(7)}\end{array}$} & $\begin{array}{c}\text { Apparently } \\
\text { healthy } \\
\text { men and } \\
\text { women }\end{array}$ & $\begin{array}{l}\text { Weight stable, } \\
\text { i.e. change in } \\
\text { BMI } \leq 0.1 \\
\text { per year }\end{array}$ & $\begin{array}{l}\text { Weight loss } \\
\text { intention } \\
\text { unspecified }\end{array}$ & & & & & $\begin{array}{l}\text { Age, BMI, systolic } \\
\text { blood pressure, } \\
\text { blood pressure } \\
\text { medication, smoking, } \\
\text { alcohol intake, } \\
\text { physical } \\
\text { activity, marital } \\
\text { status, } \\
\text { education }\end{array}$ & & & $\begin{array}{l}\text { CVD, diabetes, } \\
\text { cancer }\end{array}$ \\
\hline & $\begin{array}{l}\text { Nord-Trønde- } \\
\text { lag Health } \\
\text { Study }\end{array}$ & & $\begin{array}{l}\text { Men } \\
\text { Women }\end{array}$ & $\begin{array}{l}1319 \\
1971\end{array}$ & $\begin{array}{l}54 \cdot 3 \\
54 \cdot 0\end{array}$ & $\begin{array}{l}26.9 \mathrm{~kg} / \mathrm{m}^{2} \\
27.7 \mathrm{~kg} / \mathrm{m}^{2}\end{array}$ & $\begin{array}{l}2.2 \mathrm{~kg} / \mathrm{m}^{2} \\
2.7 \mathrm{~kg} / \mathrm{m}^{2}\end{array}$ & & $\begin{array}{l}1.6 \\
1.7\end{array}$ & $\begin{array}{l}1 \cdot 4,1 \cdot 8 \\
1 \cdot 5,2 \cdot 0\end{array}$ & \\
\hline
\end{tabular}




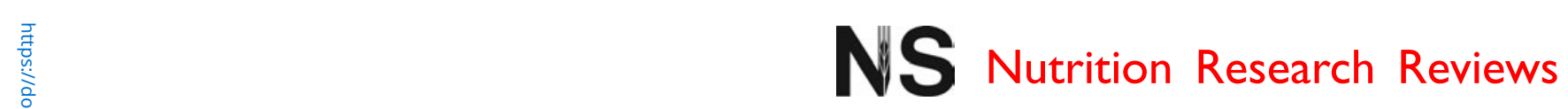

Table 1. Continued

\begin{tabular}{|c|c|c|c|c|c|c|c|c|c|c|c|}
\hline $\begin{array}{l}\text { Study } \\
\text { reference }\end{array}$ & Population & $\begin{array}{l}\text { Referent } \\
\text { group }\end{array}$ & $\begin{array}{l}\text { Weight } \\
\text { loss } \\
\text { subgroup }\end{array}$ & $\begin{array}{l}\text { Sample } \\
\text { size }(n)\end{array}$ & $\begin{array}{l}\text { Mean } \\
\text { baseline } \\
\text { age } \\
\text { and/or } \\
\text { range } \\
\text { (years) }\end{array}$ & $\begin{array}{l}\text { Baseline } \\
\text { weight } \\
\text { (kg) or } \\
\mathrm{BMI} \\
\left(\mathrm{kg} / \mathrm{m}^{2}\right)\end{array}$ & $\begin{array}{l}\text { Weight } \\
(\mathrm{kg}) \text { or } \\
\mathrm{BMI} \text { loss } \\
\left(\mathrm{kg} / \mathrm{m}^{2}\right)\end{array}$ & $\begin{array}{l}\text { Data } \\
\text { adjustments }\end{array}$ & $\begin{array}{l}\text { Relative } \\
\text { risk }\end{array}$ & $95 \% \mathrm{Cl}$ & Exclusions \\
\hline \multirow[t]{2}{*}{$\begin{array}{l}\text { Elliott et al. } \\
(2005)^{(53)}\end{array}$} & $\begin{array}{l}\text { Apparently } \\
\text { healthy } \\
\text { women }\end{array}$ & $\begin{array}{l}\text { Weight change } \\
\text { from }-1.81 \\
\text { to }+1.36 \mathrm{~kg}\end{array}$ & $\begin{array}{l}\text { Weight loss } \\
\text { intention } \\
\text { unspecified }\end{array}$ & 964 & $\begin{array}{c}42-81 \\
\text { years }\end{array}$ & - & $\begin{array}{r}116.58- \\
1.81 \mathrm{~kg}\end{array}$ & $\begin{array}{l}\text { Social class, BMI, } \\
\text { parity, smoking, } \\
\text { hormone } \\
\text { replacement } \\
\text { therapy }\end{array}$ & 0.96 & $\begin{array}{l}0.65, \\
1.43\end{array}$ & - \\
\hline & $\begin{array}{l}\text { Oral Contra- } \\
\text { ception } \\
\text { Study }\end{array}$ & & & & & & & & & & \\
\hline \multirow[t]{2}{*}{$\begin{array}{c}\text { Sørensen } \\
\text { et al. } \\
(2005)^{(8)}\end{array}$} & $\begin{array}{l}\text { Apparently } \\
\text { healthy } \\
\text { men and } \\
\text { women }\end{array}$ & Weight stable & Intentional & 398 & 41.5 & $27.4 \mathrm{~kg} / \mathrm{m}^{2}$ & $1.21 \mathrm{~kg} / \mathrm{m}^{2}$ & $\begin{array}{l}\text { Age, sex, BMI, } \\
\text { hypertension, } \\
\text { smoking, alcohol, } \\
\text { physical activity, } \\
\text { life satisfaction, } \\
\text { work status, drugs }\end{array}$ & 1.87 & $\begin{array}{l}\text { 1.22, } \\
2 \cdot 87\end{array}$ & $\begin{array}{l}\text { Angina, myocar- } \\
\text { dial } \\
\text { infarction, } \\
\text { diabetes, CVD, } \\
\text { lung disease, } \\
\text { hypertension, } \\
\text { prescription } \\
\text { drugs, } \\
\text { unemployment }\end{array}$ \\
\hline & $\begin{array}{l}\text { Finnish Twin } \\
\text { Cohort }\end{array}$ & & Unintentional & 728 & $42 \cdot 6$ & $26.72 \mathrm{~kg} / \mathrm{m}^{2}$ & $1.09 \mathrm{~kg} / \mathrm{m}^{2}$ & & 1.17 & $\begin{array}{l}0.82, \\
1.66\end{array}$ & \\
\hline \multirow[t]{4}{*}{$\begin{array}{l}\text { Wanna- } \\
\text { methee } \\
\text { et al. } \\
(2005)^{(10)}\end{array}$} & $\begin{array}{l}\text { Apparently } \\
\text { healthy } \\
\text { men }\end{array}$ & $\begin{array}{l}\text { No weight } \\
\text { change }\end{array}$ & Unintentional & 527 & $40-59$ & $25 \cdot 6 \mathrm{~kg} / \mathrm{m}^{2}$ & $3.91 \mathrm{~kg} / \mathrm{m}^{2}$ & $\begin{array}{l}\text { Age, smoking, social } \\
\text { class, physical } \\
\text { activity, } \\
\text { alcohol intake, } \\
\text { obesity, } \\
\text { perceived health } \\
\text { status, CVD, cancer, } \\
\text { hypertension, stroke }\end{array}$ & 1.71 & $\begin{array}{r}1.33, \\
2 \cdot 19\end{array}$ & - \\
\hline & $\begin{array}{l}\text { British Regional } \\
\text { Heart Study }\end{array}$ & & Intentional & 342 & $40-59$ & $28.0 \mathrm{~kg} / \mathrm{m}^{2}$ & $2.37 \mathrm{~kg} / \mathrm{m}^{2}$ & & 1.00 & $\begin{array}{l}0.91, \\
1.10\end{array}$ & \\
\hline & & & $\begin{array}{l}\text { Intentional, } \\
\text { personal } \\
\text { reason }\end{array}$ & 178 & $40-59$ & $26.9 \mathrm{~kg} / \mathrm{m}^{2}$ & $2.31 \mathrm{~kg} / \mathrm{m}^{2}$ & & 0.59 & $\begin{array}{r}0.34 \\
1.00\end{array}$ & \\
\hline & & & $\begin{array}{l}\text { Intentional, } \\
\text { physician's } \\
\text { advice }\end{array}$ & 164 & $40-59$ & $28.5 \mathrm{~kg} / \mathrm{m}^{2}$ & $2.44 \mathrm{~kg} / \mathrm{m}^{2}$ & & 1.37 & $\begin{array}{l}0.96, \\
1.94\end{array}$ & \\
\hline \multirow[t]{2}{*}{$\begin{array}{l}\text { Breeze et al. } \\
(2006)^{(54)}\end{array}$} & $\begin{array}{l}\text { Apparently } \\
\text { healthy } \\
\text { men }\end{array}$ & $\begin{array}{l}\text { Minimal weight } \\
\text { change, i.e. } \\
\text { loss } \\
0-3 \mathrm{~kg} \text { or } \\
\text { gain } 0-3 \mathrm{~kg}\end{array}$ & $\begin{array}{l}\text { Weight loss } \\
\text { intention } \\
\text { unspecified }\end{array}$ & 554 & $40-69$ & - & $\geq 10 \mathrm{~kg}$ & $\begin{array}{l}\text { Age, marital status, } \\
\text { employment, } \\
\text { smoking, respiratory } \\
\text { symptoms, heart } \\
\text { disease indicators, } \\
\text { diastolic blood } \\
\text { pressure, total } \\
\text { cholesterol }\end{array}$ & 1.88 & $1 \cdot 6,2 \cdot 2$ & - \\
\hline & $\begin{array}{l}\text { Whitehall } \\
\text { Cohort }\end{array}$ & & & 1190 & $40-69$ & - & $4-9 \mathrm{~kg}$ & & $1 \cdot 26$ & $1 \cdot 1,1 \cdot 5$ & \\
\hline \multirow[t]{2}{*}{$\begin{array}{l}\text { Nilsson et al. } \\
(2002)^{(55)}\end{array}$} & $\begin{array}{l}\text { Healthy } \\
\text { Swedish } \\
\text { men ( } n \text { 5194) }\end{array}$ & $\begin{array}{l}\text { Weight stable } \\
\left( \pm 0.1 \mathrm{~kg} / \mathrm{m}^{2}\right)\end{array}$ & $\begin{array}{l}\text { Weight loss } \\
\text { unspecified } \\
\text { (no direct } \\
\text { question) }\end{array}$ & 464 & $\begin{array}{l}47 \text { years } \\
\text { (38-52 } \\
\text { years) }\end{array}$ & $\begin{array}{r}22-25 \\
\mathrm{~kg} / \mathrm{m}^{2}\end{array}$ & - & Age & 1.39 & $\begin{array}{l}0.98, \\
1.95\end{array}$ & \multirow[t]{2}{*}{$\begin{array}{l}\text { Cancer deaths, } \\
\text { disease at } \\
\text { baseline, } \\
\text { deaths } \\
\text { in year } 1 \text { of } \\
\text { follow-up }\end{array}$} \\
\hline & & & & 482 & & $\frac{26+}{\mathrm{kg} / \mathrm{m}^{2}}$ & & & 1.71 & $\begin{array}{l}\text { 1.18, } \\
2.47\end{array}$ & \\
\hline
\end{tabular}




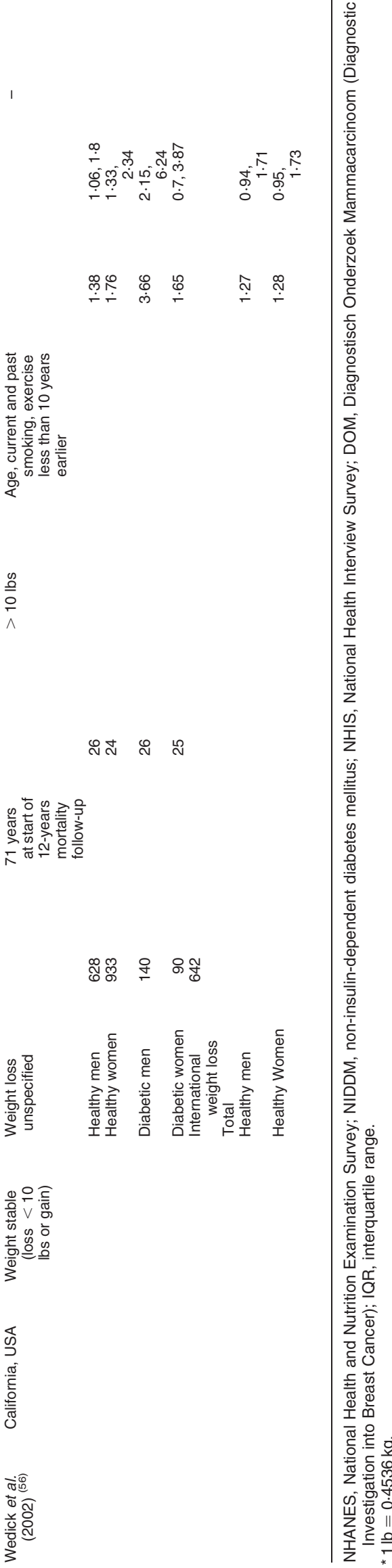

relative risk (RR) of all-cause mortality ${ }^{(13,14)}$. Methodological problems have also been identified, for example, the method by which the weight loss was achieved has usually not been reported (although dietary energy restriction is likely to have been a major factor), while weight changes before and after the recording periods have usually not been determined ${ }^{(12-16)}$.

In light of the current obesity epidemic and the resulting focus on encouraging those with BMI above $25 \mathrm{~kg} / \mathrm{m}^{2}$ to lose weight by changing their diet and lifestyle ${ }^{(19)}$, it is important to establish whether the long-term effects of weight loss benefit life expectancy. The current advice from the UK Department of Health's Obesity Care Programme is for those who are overweight or obese to reduce energy intake and increase physical activity as a method of lifestyle modification $^{(18)}$. Further treatment and advice may need to consider a broad spectrum of evidence so as not to rule out potential investigations that identify subgroups of patients, or certain conditions, where weight loss may be detrimental to health and increase mortality ${ }^{(19)}$.

The aim of the present study was to examine the available evidence of the impact of weight loss, as a lifestyle intervention, on the RR of all-cause mortality and to quantify this using meta-analysis. Data were pooled in a number of different ways in order to examine the influence of a number of possible confounders. Meta-analysis was used to provide a more objective appraisal of the evidence, integrating data from multiple prospective cohort studies to increase the power and precision of estimates of effect and reducing the likelihood of false negative results ${ }^{(20,21)}$.

\section{Methods}

Search strategy

A literature search was carried out independently by two investigators to identify prospective cohort studies that evaluated the effect of weight loss as a lifestyle intervention on mortality risk. A web search was undertaken on PubMed/Medline and ScienceDirect databases. Articles published between 1987 and 2008 and in the English language were included. Search terms included 'weight, BMI, loss, change, mortality, intentional, unintentional, relative risk, prospective and cohort'. Identified citations and abstracts were obtained from journals, libraries or authors. A hand-search of the bibliographies of retrieved papers and linked articles was also carried out.

\section{Data selection}

Inclusion criteria were prospective studies in English of adults (men and/or women) with data on body weight and weight loss over more than 1 year. Studies needed to present RR of mortality and associated $95 \%$ CI for the group that lost weight relative to a comparable reference group who lost minimal or no weight. Drug treatment studies and studies that measured weight loss following bariatric surgery were excluded, as the aim was to assess the effect of lifestyle interventions. Twenty-six publications were identified that met the inclusion criteria. Data on RR of mortality and $95 \%$ confidence limits were extracted for all 
subgroups presented by the authors (for example, men and women, intentional $v$. unintentional weight loss, obese $v$. overweight).

\section{Data analysis}

Meta-analysis was performed using Comprehensive Metaanalysis software (CMA version 2; Biostat Inc., Englewood, NJ, USA). Moderator variables such as baseline BMI (normal, overweight, obese), reason for weight loss (intentional, unintentional), baseline health status (healthy, unhealthy), method used to estimate weight loss (measured weight loss, reported weight loss) and physical activity adjustment (adjusted data, unadjusted data) were used to classify subgroups for separate analysis. For the subgroup analysis based on baseline BMI the ranges used in papers generally corresponded to those recommended by $\mathrm{WHO}^{(22)}$. Analysis was carried out using adjusted data because papers gave insufficient data on CI for unadjusted data. Although multivariable adjustment of the data varied from study to study, all adjusted for smoking. Results are shown in the form of schematic plots (Forest plots), which illustrate the size and direction of effect for each study and the weighted effect of all studies combined, with $95 \%$ (lower and upper) CI. Metaanalysis uses a weighted average of the results, in which the larger and more precise studies have more influence than the smaller ones. Results are shown for the random effects model, which assumes the underlying effect may vary for each population. This is the most appropriate model where heterogeneity is present ${ }^{(20,21)}$. Statistical significance of the overall pooled effect was based on $P<0 \cdot 05$.
Health status of group

Study name

Healthy

Healthy

Healthy

Healthy

Healthy

Healthy

Healthy

Healthy

Healthy

Healthy

Healthy

Healthy

Unhealthy

Unhealthy

Unhealthy

Unhealthy

Unhealthy

Unhealthy

Unhealthy

Overall

\begin{tabular}{|c|c|c|c|c|}
\hline French et al. (1999)(47) & $1 \cdot 18$ & 0.94 & 1.48 & 0.153 \\
\hline Gregg et al. (2003) $)^{(31)}$ & 0.76 & 0.60 & 0.97 & 0.025 \\
\hline Sørensen et al. $(2005)^{(8)}$ & 1.87 & $1 \cdot 22$ & $2 \cdot 87$ & 0.004 \\
\hline Wannamethee et al. $(2005)^{(10)}$ & 1.37 & 0.96 & 1.95 & 0.079 \\
\hline Wannamethee et al. $(2005)^{(10)}$ & 0.59 & 0.34 & 1.01 & 0.055 \\
\hline Wedick et al. (2002) ${ }^{(56)}$ & $1 \cdot 27$ & 0.94 & 1.71 & 0.118 \\
\hline Wedick et al. (2002) ${ }^{(56)}$ & $1 \cdot 28$ & 0.95 & 1.73 & 0.107 \\
\hline Williamson et al. (1995) ${ }^{(45)}$ & $1 \cdot 12$ & 0.94 & 1.33 & 0.201 \\
\hline Williamson et al. (1995) & 0.98 & 0.82 & $1 \cdot 17$ & 0.824 \\
\hline Williamson et al. (1999)(48) & 1.09 & 0.98 & $1 \cdot 21$ & 0.109 \\
\hline Williamson et al. (1999)(48) & 1.07 & 0.96 & $1 \cdot 20$ & 0.235 \\
\hline \multirow[t]{2}{*}{ Yaari \& Goldbourt (1998)(46) } & $1 \cdot 30$ & 0.94 & 1.80 & 0.116 \\
\hline & $1 \cdot 11$ & 1.00 & 1.22 & 0.050 \\
\hline Gregg et al. (2004)(51) & 0.83 & 0.63 & 1.09 & 0.175 \\
\hline Williamson et al. (1995) & 0.80 & 0.68 & 0.94 & 0.007 \\
\hline Williamson et al. (1995) & 0.81 & 0.71 & 0.92 & 0.001 \\
\hline Williamson et al. (1999)(48) & 1.01 & 0.91 & 1.12 & 0.851 \\
\hline Williamson et al. (1999)(48) & 1.02 & 0.94 & $1 \cdot 11$ & 0.641 \\
\hline \multirow[t]{3}{*}{ Williamson et al. (2000)(4) } & 0.75 & 0.67 & 0.84 & 0.000 \\
\hline & 0.87 & 0.77 & 0.99 & 0.028 \\
\hline & 1.01 & 0.93 & 1.09 & 0.892 \\
\hline
\end{tabular}

Statistics for each study

$\mathrm{RR}$ and $95 \% \mathrm{Cl}$

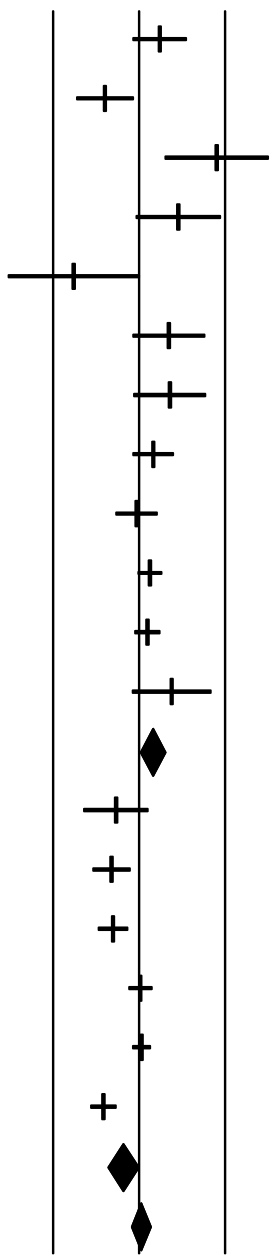

$\begin{array}{llllll}0.1 & 0.2 & 0.5 & 1 & 2 & 5\end{array}$

Favours A

Favours B

Fig. 1. Mortality risk for intentional weight loss according to health status. RR, relative risk. 


\section{Results}

\section{Study characteristics}

Table 1 shows a summary of the characteristics of the study populations and subgroups. Sample sizes ranged from 34 to 5008 subjects and the majority of the data was collected from white populations of US and UK origin. All of the studies were designed to investigate RR of mortality and weight change. The stage of life during which weight change occurred varied between adulthood, middle age and old age and the follow-up period ranged from 2 to 20 years.

\section{Quantitative data synthesis}

Owing to the acknowledged importance of whether weight loss is intended or not, results are presented for (a) intentional, (b) unintentional and (c) weight loss not specified. For the main category of interest, i.e. intentional weight loss, sub-analyses are given for healthy $v$. unhealthy subjects. These have then been further analysed to examine the influence of moderators and confounders.

\section{Intentional weight loss}

Figure 1 shows the RR of all-cause mortality in relation to intentional weight loss. Overall, there was no significant effect (RR 1.01 (95\% CI 0.93, 1.09); $P=0.89$ ). However, among healthy subjects, RR was increased $11 \%$ by weight loss (RR 1.11 (95\% CI 1.00, 1.22); $P=0.05)$, whereas it was reduced in unhealthy subjects by a similar amount (RR 0.87 (95\% CI 0.77, 0.99); $P=0.028$ ).

\section{Unintentional weight loss}

Unintentional weight loss was associated with higher mortality (RR 1.22 (95\% CI 1.09, 1.37); $P=0.001$ ) (Fig. 2), as has been shown in other studies. Unintentional weight loss is usually considered an indicator of pre-existing or silent disease and this group was not considered further.

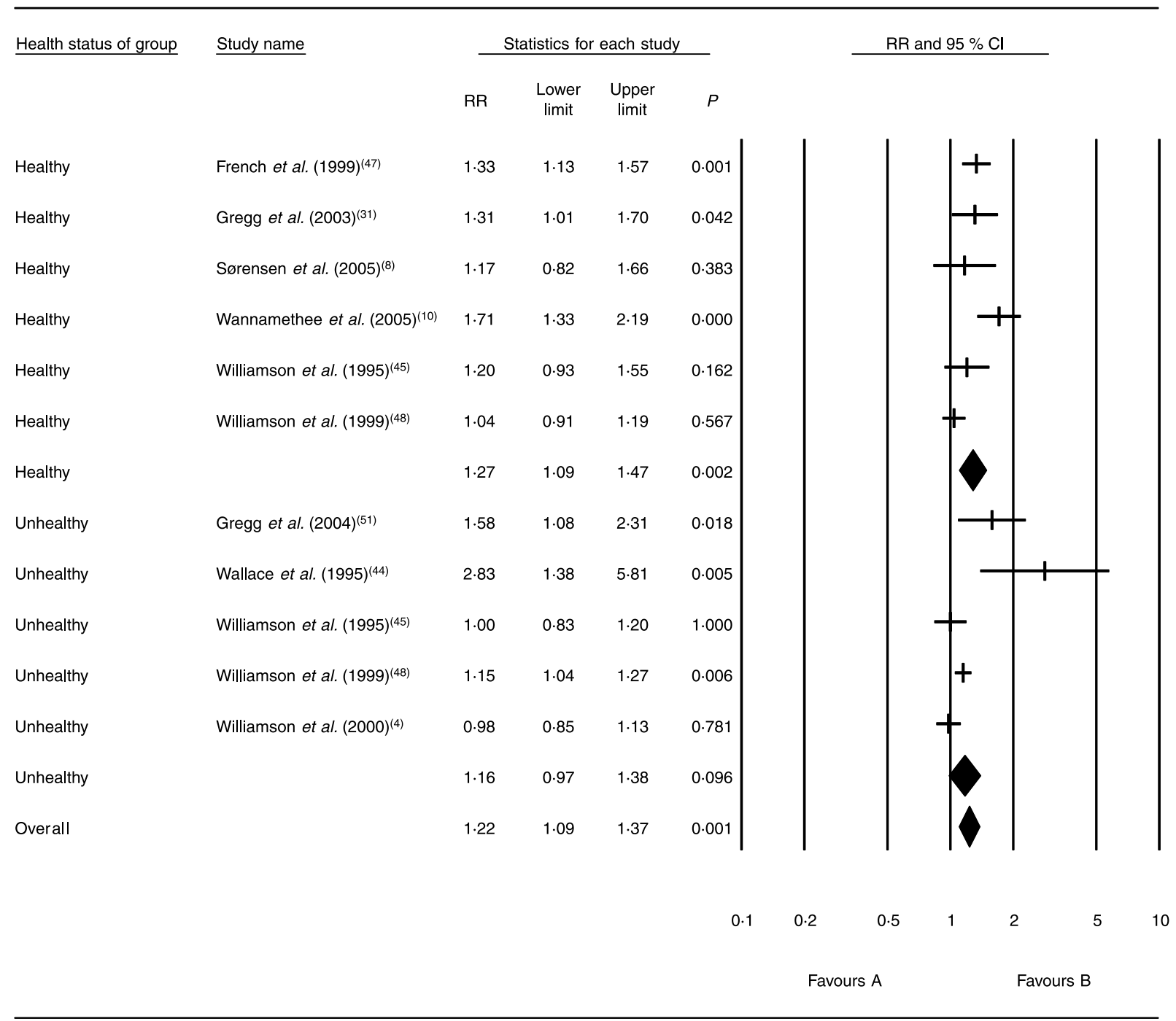

Fig. 2. Mortality risk for unintentional weight loss according to health status. RR, relative risk. 


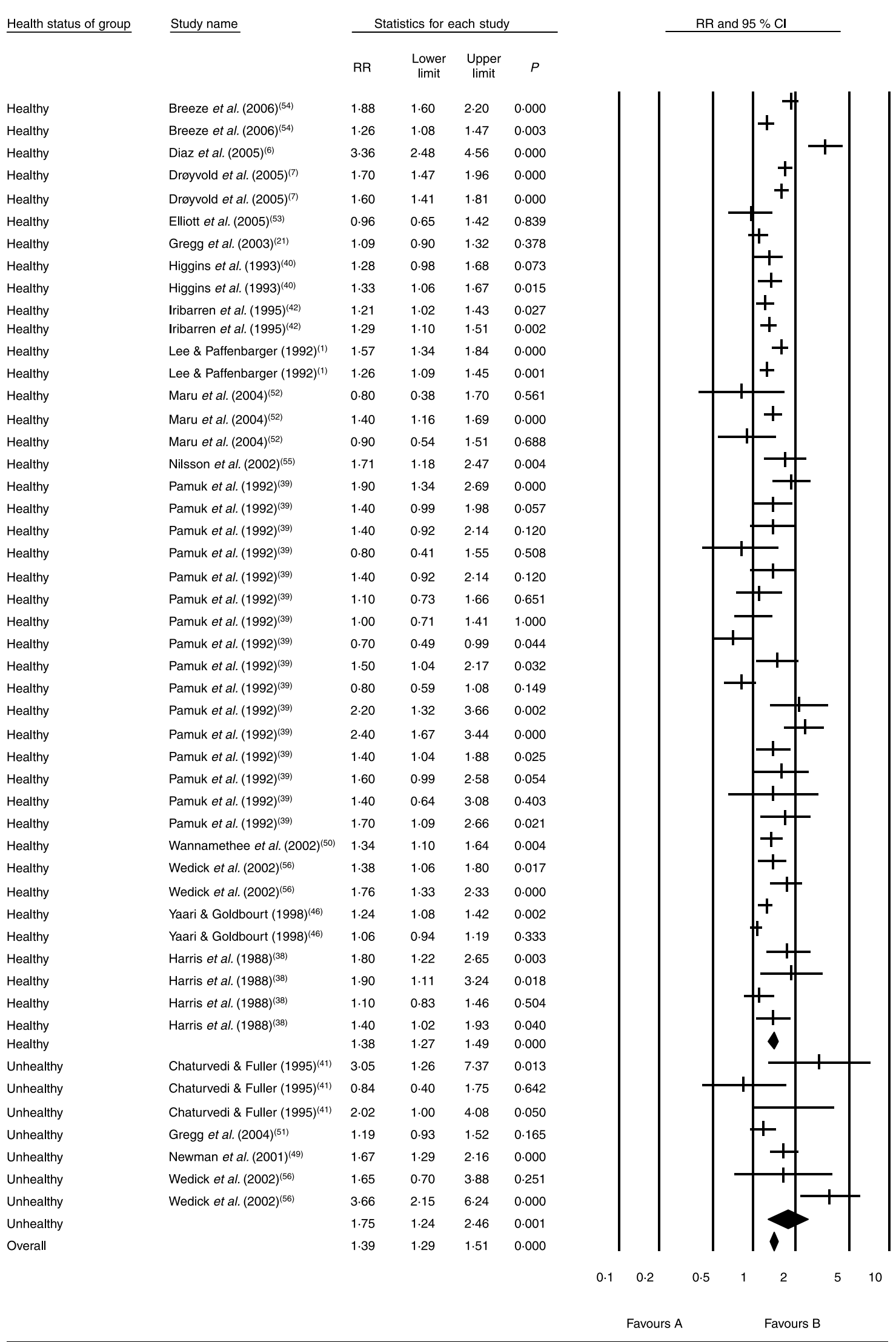

Fig. 3. Mortality risk for weight loss (intention unknown) according to health status. RR, relative risk. 


\section{Unknown or unspecified cause of weight loss}

Where the cause of weight loss was unspecified, there was also excess mortality (RR 1.39 (95\% CI 1.29, 1.51); $P<0.001$ ) (Fig. 3). Most of these studies were on 'healthy' subjects, but the subgroup who were unhealthy had even higher mortality associated with weight loss (RR $1.75(95 \%$ CI $1.24,2.46) ; P=0.001)$. Studies where weight loss intention was not explored may suffer from the same problem of confounding by illness as those in which weight loss was unintentional. The remaining analyses were all performed using studies of intentional weight loss only.

\section{Subgroup analyses of intentional weight loss}

Relative weight at baseline. Weight loss appeared to benefit obese weight losers who were also classified as unhealthy at baseline (RR 0.84 (95\% CI 0.73, 0.97); $P=0.018$ ) but had no benefit for healthy obese (RR 1.02). Overall, there was no change in risk for the obese group (RR 0.94 (95\% CI 0.86, 1.04); $P=0.002$ ) (Fig. 4). For intentional weight losers whose baseline BMI was within the normal to overweight range, or for mixed-weight populations, the RR of mortality was increased (RR 1.09 (95\% CI 1.02, 1.17); $P=0.008)$ (Fig. 5).
Method of assessing weight loss. The majority of study groups with data on intentional weight loss (fifteen out of eighteen studies) relied on reported measurements of weight or weight loss. Among these, RR associated with weight loss was near unity. However, the three study groups with actual measurement had a net RR of 1.28 (95\% CI 1.07, 1.53) (Fig. 6).

Physical activity adjustment. Adjustment for physical activity was made in most studies (fourteen out of eighteen studies) but there was essentially no difference in the RR according to whether the models had adjusted for activity or not (RR 0.98 v. 1.01 where adjusted for physical activity) (Fig. 7).

\section{Discussion \\ Main findings}

Meta-analysis was used to explore the effect of weight loss on mortality using sensitivity and subgroup analysis to explore some of the likely causes of heterogeneity, especially intentionality, health and baseline BMI. Whereas weight loss for unknown or unspecified reasons was clearly associated with excess mortality, intentional weight loss

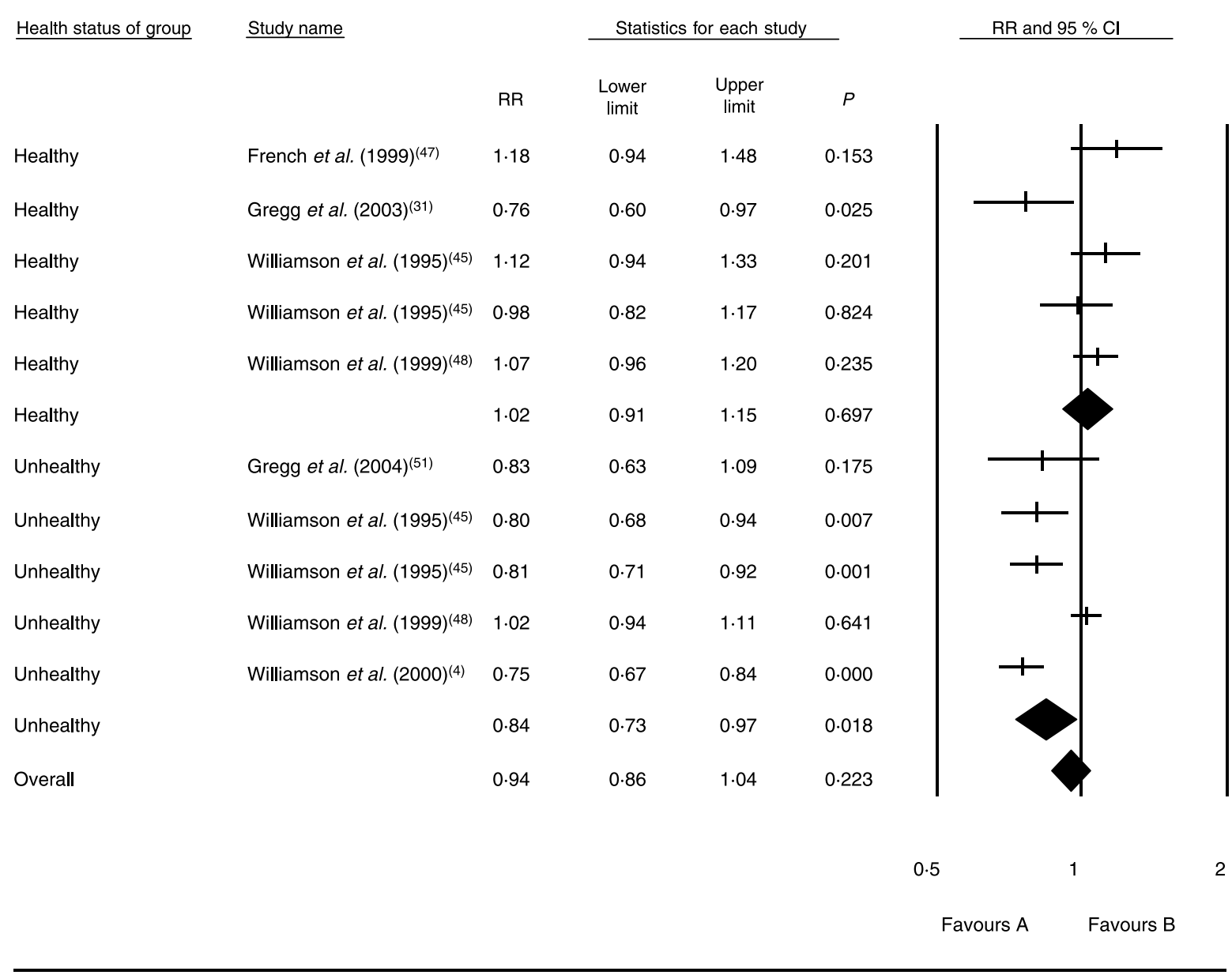

Fig. 4. Mortality risk for intentional weight loss among obese adults. RR, relative risk. 
resulted in virtually no change in mortality overall. Importantly, we found opposing effects among healthy and unhealthy adults and between the obese and those with more moderate degrees of overweight or from the general population. The excess risk of weight loss in healthy adults was estimated to be of the order of $11 \%$. This was counterbalanced by a benefit of about $13 \%$ among unhealthy adults (i.e. those with diabetes or obesity-related health conditions).

\section{Other studies}

The literature is equivocal on the risks and benefits of weight $\operatorname{loss}^{(15,16)}$. Many prospective studies and reviews appear to show an increased mortality associated with weight loss ${ }^{(12)}$, which runs counter to conventional wisdom relating to the adverse effects of obesity and the beneficial changes in risk factors associated with weight loss ${ }^{(23)}$. It has been argued that methodological weaknesses explain much of this paradox, including failure to adjust for known confounders $^{(24)}$. In particular, it has been claimed that intentionality of weight loss is key ${ }^{(16)}$ but many studies fail to distinguish between intentional and unintentional weight loss, the latter being a cardinal sign of ill health and a predictor of increased mortality in old age ${ }^{(25,26)}$.

Some clinical trials have demonstrated beneficial effects of weight loss with regard to morbidity in individuals suffering from either diabetes, obesity-related health conditions, cancer or other diseases ${ }^{(3)}$. There are also an increasing number of favourable reports from bariatric surgery, such as the 'Swedish obese subjects' (SOS) study which has shown that substantial long-term weight reduction appreciably improves the cardiovascular risk profile of morbidly obese subjects, ultimately resulting in a decrease in overall mortality ${ }^{(12)}$. Although such data may be encouraging, their success cannot necessarily be extrapolated to the public health setting where the weight losses normally achieved by diet are modest and difficult to sustain, and the subjects generally less severely obese and with few co-morbidities. Another study, due to report in 2015, will provide valuable additional data. This is the Look AHEAD (Action For Health in Diabetes) clinical trial, which is assessing the long-term effects (up to 11.5 years) of an intensive weight-loss programme delivered over 4 years in overweight and obese individuals with type 2 diabetes.

\section{Interpretation of present analysis}

In the present review and meta-analysis, intentional weight loss modestly reduced the risk of all-cause mortality only among the subgroup of unhealthy adults (by approximately $13 \%$ ), especially among those who were also obese (by approximately $16 \%$ ). All these studies relied on reported estimates of body weight. Self-reporting of body weight may be cheap and easily carried out; however, it is affected by a number of biases. Actual measurement of body weight using appropriate devices is recommended for complete accuracy and reliability of the data ${ }^{(27)}$.

Our finding of a marginally increased risk of death among overweight but otherwise healthy adults who lost weight intentionally, if true, has important public health implications. This observation is consistent with recent findings using National Health and Nutrition Examination Survey

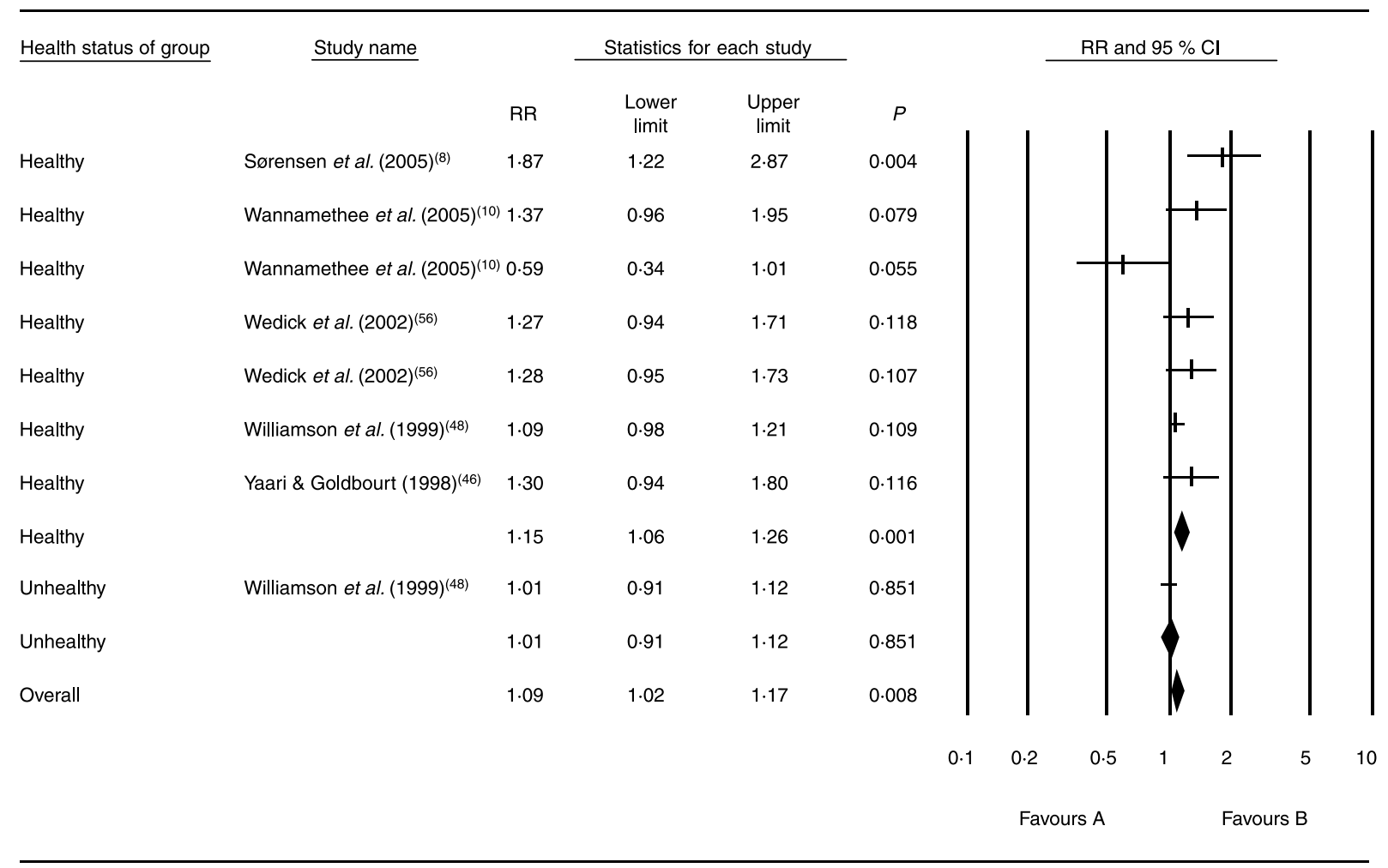

Fig. 5. Mortality risk for intentional weight loss among overweight or mixed populations. RR, relative risk. 
(NHANES) data that showed that the ideal weight for longevity was the overweight category, or BMI 23$30 \mathrm{~kg} / \mathrm{m}^{2(28-30)}$.

Why should intentional weight loss have opposing effects in different groups of individuals? One possibility is that obese individuals with risk factors may show a benefit because they are more motivated to make a series of changes such as reducing fat intake or increasing exercise level, and these may lower RR of mortality by benefiting overall health status $^{(31)}$. Unhealthy individuals are also more likely to be recipients of health care and medical interventions. It is more difficult to explain why intentional weight loss should have an adverse effect among healthy but overweight individuals. More data on method of weight loss, persistence of weight loss and body composition would be helpful in this regard. Weight loss via energy restriction may do little to alter the relative distribution of body fat and may result in decreased lean body mass. A reanalysis of the Framingham Heart Study and the Tecumseh Community Study suggests that weight loss as a result of a reduction in body fat may reduce all-cause mortality while weight loss as a result of a reduction in lean body mass may increase it ${ }^{(32)}$. Given the significance of fat distribution and the lean body mass:fat ratio in health prognosis ${ }^{(33)}$, it is imperative that future studies attempt to measure more than just weight or BMI. Furthermore, studies must adequately disentangle the influence of physical activity and/or fitness, which may influence both body weight and the morbidity and mortality outcomes under study. Most studies did not include assessment of physical activity and those that did used questionnaires rather than physical fitness, which is a stronger predictor of mortality ${ }^{(34,35)}$. The focus of new research may most usefully be directed to examining survival among those population groups that might be expected to benefit most from weight loss. These include those with diabetes, those with obesity-related conditions (such as hypertension) and certain ethnic groups.

\section{Limitations}

The present study inevitably has some limitations. The literature search was carried out using only two databases, but was complemented by thorough checking of crossreferences and inclusion of new reviews published in 2008. Limitations of the evidence base include the fact that none of the studies provided information on the method of weight loss, which is relevant because it is not clear if weight loss through energy restriction or increased energy expenditure differentially influences long-term outcomes. Second, weight loss was usually assessed retrospectively and subjectively, often at two time points some distance removed from the ultimate outcome, i.e.

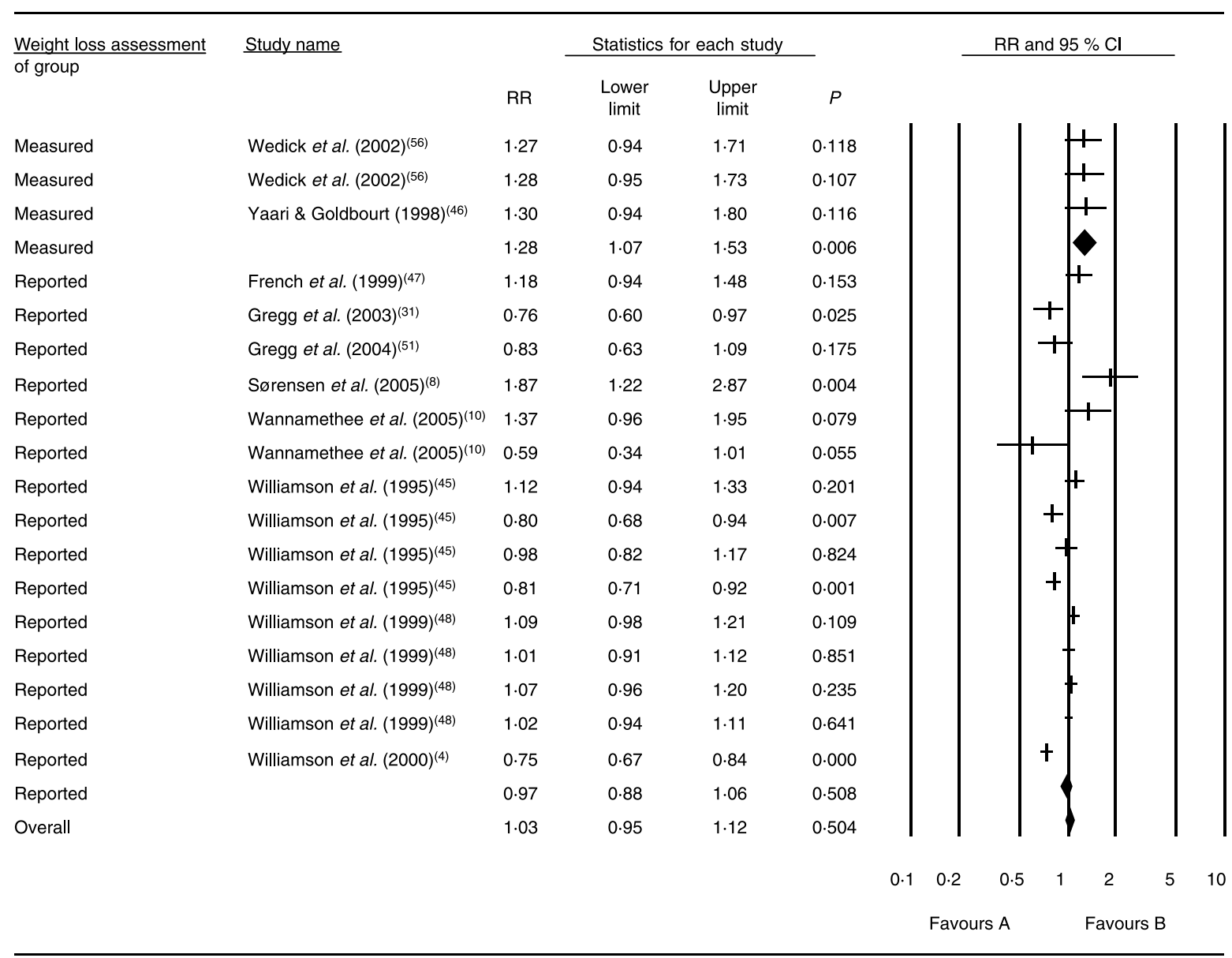

Fig. 6. Mortality risk for intentional weight loss according to weight loss assessment method. RR, relative risk. 


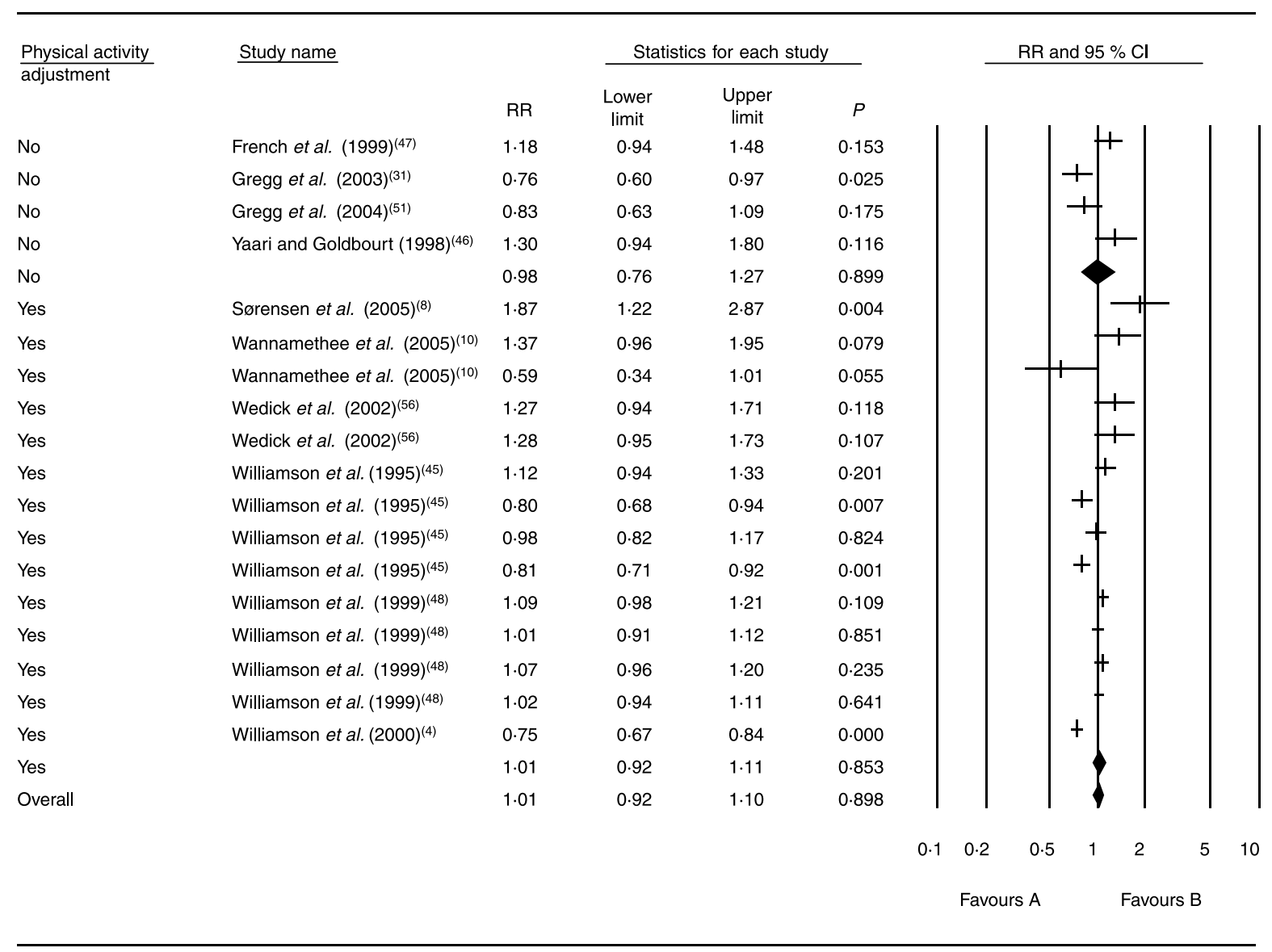

Fig. 7. Mortality risk for intentional weight loss according to adjustment for physical activity. RR, relative risk.

death. It is thus difficult to be sure that the weight loss estimate does not represent a transitory phase and that it is representative of a reasonable period of adult life. Third, the studies differed in the statistical treatment of covariates or confounders in adjusted models (for example, some excluded smokers, others adjusted for smoking). These problems are common to all attempts to review and pool data from different studies, and the present results are consistent with other recent reviews that have not used meta-analysis ${ }^{(16,36)}$. Furthermore, using a meta-analysis stratified by intentionality, health and baseline BMI, we were able to quantify effect sizes in different groups. The robustness of intentionality measures has been questioned ${ }^{(16)}$ because it depends on the question asked and may change during the course of the followup $^{(37)}$. The study by Sørensen et al. ${ }^{(8)}$ was unusual in assessing intentionality prospectively and also reported the largest effect size (RR 1.87) ${ }^{(8)}$. However, as it was of high quality (as judged by Simonsen et al. ${ }^{(16)}$ ), we did not consider its exclusion justified in the main analysis. Instead, sensitivity analysis showed that the effect of excluding this paper would be to reduce the RR from 1.11 to 1.09 . On balance we think it unlikely that our estimates of higher risk are inflated, since most sources of misclassification and measurement error would tend to result in underestimation of effect (for example, selfreported body weight).

\section{Conclusion}

Recently a great emphasis has been placed on weight loss by lifestyle change for everyone who is, even slightly, overweight. However, a review of the available literature, complemented by meta-analysis, suggests that at-risk individuals may benefit, but for healthy overweight individuals intentional weight loss does not decrease mortality and may even increase it. Appropriately designed intervention studies in subgroups differing by age, sex and ethnic group, as well as by risk status, are urgently needed. Until more reliable data are available to demonstrate consistent improvements in survival, the question remains as to whether the correction of obesity per se should have such emphasis as a clinical and public health target.

\section{Acknowledgements}

We wish to thank Orlaith McDaid for her contribution to the preparation of this paper.

The present review was funded by The World Sugar Research Organisation. The findings and conclusions are those of the authors and do not necessarily represent the views of the funding organisation.

The authors have no conflicts of interest to declare. 


\section{References}

1. Lee IM \& Paffenbarger RS Jr (1992) Change in body weight and longevity. JAMA 268, 2045-2049.

2. Van Gaal LF, Wauters MA \& De Leeuw IH (1997) The beneficial effects of modest weight loss on cardiovascular risk factors. Int J Obes Relat Metab Disord 21, Suppl. 1, S5-S9.

3. Goldstein DJ (1992) Beneficial health effects of modest weight loss. Int J Obes Relat Metab Disord 16, 397-415.

4. Williamson DF, Thompson TJ, Thun M, et al. (2000) Intentional weight loss and mortality among overweight individuals with diabetes. Diabetes Care 23, 1499-1504.

5. Sjöström L, Narbro K, Sjöström CD, et al. (2007) Effects of bariatric surgery on mortality in Swedish obese subjects. $N$ Engl J Med 357, 741-752.

6. Diaz VA, Mainous AG III \& Everett CJ (2005) The association between weight fluctuation and mortality: results from a population-based cohort study. J Community Health 30, $153-165$

7. Drøyvold WB, Lund Nilsen TI, Lydersen S, et al. (2005) Weight change and mortality: the Nord-Trøndelag Health Study. J Intern Med 257, 338-345.

8. Sørensen TI, Rissanen A, Korkeila M, et al. (2005) Intention to lose weight, weight changes, and 18-y mortality in overweight individuals without co-morbidities. PLoS Med 2, e171.

9. Sauvaget C, Ramadas K, Thomas G, et al. (2008) Body mass index, weight change and mortality risk in a prospective study in India. Int J Epidemiol 37, 990-1004.

10. Wannamethee SG, Shaper AG \& Lennon L (2005) Reasons for intentional weight loss, unintentional weight loss, and mortality in older men. Arch Intern Med 165, 1035-1040.

11. Eilat-Adar S, Goldbourt U, Resnick HE, et al. (2005) Intentional weight loss, blood lipids and coronary morbidity and mortality. Curr Opin Lipidol 16, 5-9.

12. Nilsson PM (2008) Is weight loss beneficial for reduction of morbidity and mortality? What is the controversy about? Diabetes Care 31, Suppl. 2, S278-S283.

13. Sørensen TI (2003) Weight loss causes increased mortality: pros. Obes Rev 4, 3-7.

14. Yang D, Fontaine KR, Wang C, et al. (2003) Weight loss causes increased mortality: cons. Obes Rev 4, 9-16.

15. Poobalan AS, Aucott LS, Smith WC, et al. (2007) Long-term weight loss effects on all cause mortality in overweight/obese populations. Obes Rev 8, 503-513.

16. Simonsen MK, Hundrup YA, Obel EB, et al. (2008) Intentional weight loss and mortality among initially healthy men and women. Nutr Rev 66, 375-386.

17. World Health Organization \& Food and Agriculture Organization (2003) Diet, Nutrition and the Prevention of Chronic Diseases. Geneva: WHO.

18. Department of Health (2006) Your Weight, Your Health. London: Department of Health Central Office of Information. http://www.dh.gov.uk/en/Publicationsandstatistics/Publications/PublicationsPolicyAndGuidance/DH_4134408

19. Astrup A (2003) Weight loss and increased mortality: epidemiologists blinded by observations? Obes Rev 4, 1-2.

20. Egger M \& Smith GD (1997) Meta-analysis: potentials and promise. BMJ 315, 1371-1374.

21. Egger M, Smith GD \& Phillips AN (1997) Meta-analysis: principles and procedures. BMJ 315, 1533-1537.

22. World Health Organization (2000) Obesity: Preventing and Managing the Global Epidemic. Geneva: WHO.

23. Heitmann BL, Svendsen OL, Martinussen T, et al. (1997) Significance of intentional weight loss on health (article in Danish). Ugeskr Laeger 159, 4099-4104.
24. Stampfer M (2005) Weight loss and mortality: what does the evidence show? PLoS Med 2, e181.

25. Ryan C, Bryant E, Eleazer P, et al. (1995) Unintentional weight loss in long-term care: predictor of mortality in the elderly. South Med J 88, 721-724.

26. Shahar A, Shahar D, Kahar Y, et al. (2005) Low-weight and weight loss as predictors of morbidity and mortality in old age (article in Hebrew). Harefuah 144, 443-448, 452.

27. John U, Hanke M, Grothues J, et al. (2006) Validity of overweight and obesity in a nation based on self-report versus measurement device data. Eur J Clin Nutr 60, 372-377.

28. Fontaine KR, Redden DT, Wang C, et al. (2003) Years of life lost due to obesity. JAMA 289, 187-193.

29. Flegal KM, Graubard BI, Williamson DF, et al. (2005) Excess deaths associated with underweight, overweight, and obesity. JAMA 293, 1861-1867.

30. Flegal KM, Graubard BI, Williamson DF, et al. (2007) Cause-specific excess deaths associated with underweight, overweight, and obesity. JAMA 298, 2028-2037.

31. Gregg EW, Gerzoff RB, Thompson TJ, et al. (2003) Intentional weight loss and death in overweight and obese U.S. adults 35 years of age and older. Ann Intern Med 138, $383-389$.

32. Allison DB, Zannolli R, Faith MS, et al. (1999) Weight loss increases and fat loss decreases all-cause mortality rate: results from two independent cohort studies. Int J Obes Relat Metab Disord 23, 603-611.

33. Berentzen T \& Sørensen TI (2006) Effects of intended weight loss on morbidity and mortality: possible explanations of controversial results. Nutr Rev 64, 502-507.

34. Warburton DE, Nicol CW \& Bredin SS (2006) Health benefits of physical activity: the evidence. CMAJ 174, $801-809$.

35. Myers J, Kaykha A, George S, et al. (2004) Fitness versus physical activity patterns in predicting mortality in men. Am J Med 117, 912-918.

36. Fontaine KR \& Allison DB (2001) Does intentional weight loss affect mortality rate? Eat Behav 2, 87-95.

37. Coffey CS, Gadbury GL, Fontaine KR, et al. (2005) The effects of intentional weight loss as a latent variable problem. Stat Med 24, 941-954.

38. Harris T, Cook EF, Garrison R, et al. (1988) Body mass index and mortality among nonsmoking older persons. The Framingham Heart Study. JAMA 259, 1520-1524.

39. Pamuk ER, Williamson DF, Madans J, et al. (1992) Weight loss and mortality in a national cohort of adults, 1971-1987. Am J Epidemiol 136, 686-697.

40. Higgins M, D’Agostino R, Kannel W, et al. (1993) Benefits and adverse effects of weight loss. Observations from the Framingham Study. Ann Intern Med 119, 758-763.

41. Chaturvedi N \& Fuller JH (1995) Mortality risk by body weight and weight change in people with NIDDM. The WHO Multinational Study of Vascular Disease in Diabetes. Diabetes Care 18, 766-774.

42. Iribarren C, Sharp DS, Burchfiel CM, et al. (1995) Association of weight loss and weight fluctuation with mortality among Japanese American men. $N$ Engl J Med 333, 686-692.

43. Manson JE, Willett WC, Stampfer MJ, et al. (1995) Body weight and mortality among women. $N$ Engl $J$ Med 333, $677-685$.

44. Wallace JI, Schwartz RS, LaCroix AZ, et al. (1995) Involuntary weight loss in older outpatients: incidence and clinical significance. J Am Geriatr Soc 43, 329-337.

45. Williamson DF, Pamuk E, Thun M, et al. (1995) Prospective study of intentional weight loss and mortality 
in never-smoking overweight US white women aged 40-64 years. Am J Epidemiol 141, 1128-1141.

46. Yaari S \& Goldbourt U (1998) Voluntary and involuntary weight loss: associations with long term mortality in 9,228 middle-aged and elderly men. Am J Epidemiol 148, 546-555.

47. French SA, Folsom AR, Jeffery RW, et al. (1999) Prospective study of intentionality of weight loss and mortality in older women: the Iowa Women's Health Study. Am J Epidemiol 149, 504-514.

48. Williamson DF, Pamuk E, Thun M, et al. (1999) Prospective study of intentional weight loss and mortality in overweight white men aged 40-64 years. Am J Epidemiol 149, 491-503.

49. Newman AB, Yanez D, Harris T, et al. (2001) Weight change in old age and its association with mortality. J Am Geriatr Soc 49, 1309-1318.

50. Wannamethee SG, Shaper AG \& Walker M (2002) Weight change, weight fluctuation, and mortality. Arch Intern Med 162, 2575-2580.

51. Gregg EW, Gerzoff RB, Thompson TJ, et al. (2004) Trying to lose weight, losing weight, and 9-year mortality in overweight U.S. adults with diabetes. Diabetes Care 27, 657-662.

52. Maru S, van der Schouw YT, Gimbrère CH, et al. (2004) Body mass index and short-term weight change in relation to mortality in Dutch women after age 50 y. Am J Clin Nutr 80, 231-236.

53. Elliott AM, Aucott LS, Hannaford PC, et al. (2005) Weight change in adult life and health outcomes. Obes Res 13, 1784-1792.

54. Breeze E, Clarke R, Shipley MJ, et al. (2006) Cause-specific mortality in old age in relation to body mass index in middle age and in old age: follow-up of the Whitehall cohort of male civil servants. Int J Epidemiol 35, 169-178.

55. Nilsson PM, Nilsson JA, Hedblad B, et al. (2002) The enigma of increased non-cancer mortality after weight loss in healthy men who are overweight or obese. J Intern Med 252, 70-78.

56. Wedick NM, Barrett-Connor E, Knoke JD, et al. (2002) The relationship between weight loss and all-cause mortality in older men and women with and without diabetes mellitus: the Rancho Bernado study. J Am Geriatr Soc 50, 1810-1815. 